\title{
Erratum to: Optimal Design of Reinforced Concrete Cantilever Retaining Walls Considering the Requirement of Slope Stability
}

\author{
Boonchai Ukritchon*, Sophea Chea**, and Suraparb Keawsawasvong***
}

Received September 21, 2016/Revised November 19, 2016/Accepted November 26, 2016/Published Online February 3, 2017

\section{Erratum to: KSCE Journal of Civil Engineering (2017) 21(7):2673-2682}

DOI 10.1007/s12205-017-1627-1

This erratum is published to notify a spelling error in authors' address. Please take note that changes have been made to authors, Boonchai Ukritchon* and Suraparb *** as below.

\section{Errata:}

The original version of this article:

*Associate Professor, Geotechnical Research Unit, Dept. of Civil Engineer, Faculty of Engineering, Chulalongkorn University, Bongkok 10330, Thailand (Corresponding Author, E-mail: boonchai.uk@gmail.com)

***Ph.D. Research Fellow, Geotechnical Research Unit, Dept. of Civil Engineering, Faculty of Engineering, Chulalongkorn University, Bongkok 10330, Thailand (E-mail: suraparb@hotmail.com)

\section{Was altered as:}

*Associate Professor, Geotechnical Research Unit, Dept. of Civil Engineer, Faculty of Engineering, Chulalongkorn University, Bangkok 10330, Thailand (Corresponding Author, E-mail: boonchai.uk@gmail.com)

***Ph.D. Research Fellow, Geotechnical Research Unit, Dept. of Civil Engineering, Faculty of Engineering, Chulalongkorn University, Bangkok 10330, Thailand (E-mail: suraparb@hotmail.com)

The original article has been corrected.

\footnotetext{
*Associate Professor, Geotechnical Research Unit, Dept. of Civil Engineer, Faculty of Engineering, Chulalongkorn University, Bangkok 10330, Thailand (Corresponding Author, E-mail: boonchai.uk@gmail.com)

**Technical Engineering, Cana Sino Construction Corporation, Cambodia (E-mail: chea.sp@gmail.com)

***Ph.D. Research Fellow, Geotechnical Research Unit, Dept. of Civil Engineering, Faculty of Engineering, Chulalongkorn University, Bangkok 10330, Thailand (E-mail: suraparb@hotmail.com)
} 